\title{
Spectrum, Temporal Structure, and Fluctuations in a High-Gain Free-Electron Laser Starting from Noise
}

\author{
R. Bonifacio, ${ }^{1,2}$ L. De Salvo, ${ }^{1}$ P. Pierini, ${ }^{2}$ N. Piovella,,${ }^{1}$ and C. Pellegrini ${ }^{3}$ \\ ${ }^{1}$ Dipartimento di Fisica dell'Università di Milano, Via Celoria 16, 20133 Milano, Italy \\ ${ }^{2}$ Istituto Nazionale di Fisica Nucleare-Sezione di Milano, Via Celoria 16, 20133 Milano, Italy \\ ${ }^{3}$ Department of Physics, University of California Los Angeles, 405 Hilgard Avenue, Los Angeles, California 90024
}

(Received 14 July 1993)

\begin{abstract}
We study the time structure, the frequency composition, and the shot to shot fluctuations of the radiation emitted by a free-electron laser starting from shot noise in the electron beam longitudinal distribution, taking into account slippage and finite bunch length effects. We find a very different behavior when the bunch length, $\ell_{b}$, is much longer than the cooperation length, $\ell_{c}$, or of the order of a few $\ell_{c}$. The field evolution is dominated by slippage effects in both cases, and shows the presence of superradiant spikes.
\end{abstract}

PACS numbers: $41.60 . \mathrm{Cr}, 42.60 . \mathrm{Jf}$

In this paper we study the evolution of the radiation intensity and the spectral characteristics of a high-gain free-electron laser operating in the self-amplified spontaneous emission mode (SASE) [1], i.e., starting from noise in the initial electron beam longitudinal density distribution. This problem is important for the development of extreme UV FELs, such as the soft x-ray FEL proposed recently by a SLAC-UCLA-LBL-LLNL Collaboration [2]. This evolution determines the undulator length needed to reach saturation, and also the characteristic spectral width, intensity, and intensity fluctuations. These are important parameters, determining the possibility of using the FEL in a new wavelength region, for experiments such as single shot $x$-ray imaging of biological samples.

The FEL startup from noise has been studied before [3-6] in the limit of infinitely long bunches. Here we generalize the 1D theory to include "short" and "long" bunches effects, introducing as the important parameter the ratio between the bunch length, $\ell_{b}$, and the cooperation length, $\ell_{c}$.

The problem under study is characterized by several characteristic scale lengths: the electron bunch length, the gain length, and the cooperation length. An important parameter determining the evolution of the system is the ratio of $\ell_{b}$ to $\ell_{c}$. When this ratio is larger than $2 \pi$, long bunch case, we recover the results of the previous authors for the undulator saturation length and linewidth, and we also have new results: the evaluation of the saturation length fluctuations and the intensity fluctuations, as well as the study of the temporal and frequency structure of the radiated pulse. In particular we show that although the linewidth at saturation is of the order of the FEL parameter, $\rho$, or the inverse of the number of undulator periods $[3,5]$, the radiation pulse contains many spikes, each one having a maximum duration corresponding to about $2 \pi \ell_{c}$, with large intensity fluctuations. If the ratio of $\ell_{b}$ to $\ell_{c}$ is smaller than $2 \pi$, short bunch case, the saturation length tends to be somewhat longer than in the long bunch case. One single radiation pulse is present in this case, with no inner spikes.
The fluctuations levels are much larger than in the long bunch case. In both cases one can observe superradiant behavior for sufficiently long undulators.

We consider here the one dimensional linear model in the universal scaling of Refs. $[7,8]$,

$$
\frac{\partial A}{\partial \bar{z}}+\frac{\partial A}{\partial \bar{z}_{1}}=b, \quad \frac{\partial^{2} b}{\partial \bar{z}^{2}}=i A .
$$

In these equations $\bar{z}=z / \ell_{g}, \bar{z}_{1}=-c\left(t-z / v_{\|}\right) / \ell_{c}$, where $z$ is the coordinate along the undulator axis, $\bar{z}_{1}$ is the scaled position along the bunch, $v_{\|}$is the electron velocity in the $z$ direction, and $\ell_{g}=\lambda_{w} / 4 \pi \rho$ and $\ell_{c}=\lambda_{s} / 4 \pi \rho$ are the gain length and the cooperation length, respectively. The other quantities are $\lambda_{w}$, wiggler period; $\lambda_{s}=2 \pi / k_{s}$, radiation wavelength which satisfies the resonance relation $k_{s}=2 k_{w} \gamma_{0}^{2} /\left(1+a_{w}^{2}\right)$; $A=E_{0} /\left(4 \pi m c^{2} \gamma_{0} n_{e} \rho\right)^{1 / 2}$, dimensionless field amplitude, with $m c^{2} \gamma_{0}$, average initial electron energy, $n_{e}=I / e c \sigma$, beam density, $I$, electron current, $\sigma$, beam cross section; $b=N_{\lambda}^{-1} \sum_{j=1}^{N_{\lambda}} \exp \left(-i \theta_{j}\right)$, bunching parameter, with $\theta_{j}=\left(k_{w}+k_{s}\right) z-c k_{s} t_{j}$, electron phase, and $N_{\lambda}$, number of electrons within a longitudinal distance equal to $\lambda_{s} ; \rho=\left(1 / \gamma_{0}\right)\left(a_{w} \omega_{p} / 4 c k_{w}\right)^{2 / 3}$, fundamental FEL parameter [1], with $\omega_{p}=\left(4 \pi e^{2} n_{\mathrm{e}} / m\right)^{1 / 2}$, plasma frequency and $a_{w}=e B_{w} / m c^{2} k_{w}$, wiggler parameter. Equations (1) have been obtained linearizing the Compton FEL equations [1], around the equilibrium condition with no field, unbunched, uniform density and monoenergetic electron beam, and describe the exponential growth of the signal in the high-gain regime before saturation.

We solve the system to describe the onset of the FEL process from random noise on the phases $b_{0}\left(\bar{z}_{1}\right)$ of a monoenergetic electron beam injected in the undulator. For a flattop electron beam of finite length $\bar{\ell}_{b}=\ell_{b} / \ell_{c}$, with the trailing edge at $\bar{z}_{1}=0$, the solution of Eqs. (1) is given by

$$
A\left(\bar{z}, \bar{z}_{1}\right)=\int_{0}^{\bar{z}_{1}} d \xi b_{0}\left(\bar{z}_{1}-\xi\right) G(\bar{z}, \xi)
$$

inside the beam $\left(0<\bar{z}_{1}<\bar{\ell}_{b}\right)$, and 


$$
A\left(\bar{z}, \bar{z}_{1}\right)=\int_{0}^{\bar{\ell}_{b}} d \xi b_{0}\left(\bar{\ell}_{b}-\xi\right) G\left(\bar{z}+\bar{\ell}_{b}-\bar{z}_{1}, \xi\right)
$$

in front of the beam $\left(\bar{\ell}_{b}<\bar{z}_{1}<\bar{\ell}_{b}+\bar{z}\right)$. The solution (3) is simply the inner solution (2) propagated outside of the leading edge of the bunch.

The solution is a convolution between the initial perturbation $b_{0}\left(\bar{z}_{1}\right)$ and the Green function $G\left(\bar{z}, \bar{z}_{1}\right)$, which vanishes for $\bar{z}_{1}>\bar{z}$ and whose Fourier transform is given by

$$
\tilde{G}(\bar{z}, \bar{\omega})=-i \sum_{j=1}^{3} \frac{e^{i \lambda_{j}(\bar{\omega}) \bar{z}}}{3 \lambda_{j}(\bar{\omega})-2 \bar{\omega}},
$$

and $\lambda_{j}(\bar{\omega}), j=1,2,3$, are the solutions of the cubic equation $\lambda^{3}-\bar{\omega} \lambda^{2}+1=0$, where $\bar{\omega}=\left(k-k_{s}\right) / 2 \rho k_{s}$. In the limit of a beam much longer than the slippage between the radiation and the electrons in the wiggler, $\bar{\ell}_{b} \gg \bar{z}$ (i.e., $\ell_{b} \gg N_{w} \lambda_{s}$ ), neglecting the radiation escaped from the leading edge of the beam and the slippage region at the trailing edge, we can write an approximate expression for the emitted radiation spectrum $[3,4]$ :

$$
\tilde{A}(\bar{z}, \bar{\omega})=\tilde{b}_{0}(\bar{\omega}) \tilde{G}(\bar{z}, \bar{\omega}),
$$

where $\tilde{b}_{0}(\bar{\omega})$ is the Fourier transform of the initial bunching $b_{0}\left(\bar{z}_{1}\right)$, which describes the SASE regime [4].

The function $G\left(\bar{z}, \bar{z}_{1}\right)$ can be evaluated asymptotically, for $0<\bar{z}_{1}<\bar{z}$, as $|G|^{2} \simeq \frac{1}{12 \pi(y / 2)^{2 / 3}} e^{3 \sqrt{3}(y / 2)^{2 / 3}}$, where $y=\sqrt{\bar{z}_{1}}\left(\bar{z}-\bar{z}_{1}\right)[7,8]$. The maximum of this expression, as a function of $\bar{z}_{1}$, occurs at $\bar{z}_{1}=\bar{z} / 3$, where $y$ is maximum, and its width is proportional to $\sqrt{\bar{z}}$. This solution describes a wave packet moving at a constant velocity $v_{s}=3 v_{\|} /\left(2+v_{\|} / c\right)$, with a peak growing as $(1 / 4 \pi \bar{z}) \exp (\sqrt{3} \bar{z})$. From expression (2) it is possible to demonstrate the superradiant nature of the spikes growing from the noise nonuniformities on the bunch. We specialize $b_{0}\left(\bar{z}_{1}\right)$ for the case of a short (compared to $\ell_{c}$ ) initial perturbation on the bunch of width $\bar{\Delta}$ (in units $\ell_{c}$ ), of average value $b_{0}$. Expression (2) leads to $A\left(\bar{z}, \bar{z}_{1}\right)=b_{0} \bar{\Delta} G\left(\bar{z}, \bar{z}_{1}\right)$, hence $\left|A\left(\bar{z}, \bar{z}_{1}\right)\right|^{2} \propto \bar{\Delta}^{2} \propto \rho^{2}$, that is, the emitted intensity from the short perturbation is superradiant [9]. In the case of a more complex structure of the initial noise, the field will be a superposition of superradiant spikes.

In the high-gain limit, $\bar{z} \gg 1$, the root of the cubic dispersion relation with negative imaginary part gives rise to an exponential growing spectral intensity proportional to $\exp [2|\operatorname{Im} \lambda(\bar{\omega})| \bar{z}]$. The imaginary part of the complex root $|\operatorname{Im} \lambda(\bar{\omega})|$ is zero for $\bar{\omega}>(27 / 4)^{1 / 3} \simeq 1.89$; it can be approximated as $|\operatorname{Im} \lambda| \simeq \sqrt{3} / 2\left(1-\bar{\omega}^{2} / 9\right)$ near resonance $(\bar{\omega}=0)$ and it decreases as $1 / \sqrt{|\bar{\omega}|}$ for large negative values of $\bar{\omega}$. Studying the behavior of the spectrum near $\bar{\omega}=0$, we can write $|\tilde{G}(\bar{z}, \bar{\omega})|^{2} \simeq \frac{1}{9} \exp \left\{\sqrt{3} \bar{z}-\bar{\omega}^{2} / 2 \bar{\sigma}^{2}\right\}$, where $\bar{\sigma}^{2}=9 / 2 \sqrt{3} \bar{z}$ : hence, the FWHM of the envelope of the observed spectrum for undulator longer than one gain length $\ell_{g}$ shrinks as $1 / \sqrt{\bar{z}}$ along the wiggler $[3,4]$.
We consider now the SASE regime, with the emission starting from the noise term $b_{0}\left(\bar{z}_{1}\right)=$ $N_{\lambda}^{-1} \sum_{j=1}^{N_{\lambda}} \exp \left\{-i \theta_{j 0}\right\}$, where $\theta_{j 0}$ are the random electron phases at the entrance of the wiggler. The average emitted power, in dimensionless units, is defined as

$$
\begin{aligned}
E_{L}(\bar{z}) & \equiv \frac{1}{\bar{\ell}_{b}} \int_{0}^{\bar{\ell}_{b}+\bar{z}} d \bar{z}_{1}\left\langle|A|^{2}\right\rangle=\frac{1}{\bar{\ell}_{b}} \int_{-\infty}^{\infty} d \bar{\omega}\left\langle|\tilde{A}|^{2}\right\rangle \\
& \approx \frac{1}{\bar{\ell}_{b}} \int_{-\infty}^{\infty} d \bar{\omega}\left\langle\left|\tilde{b}_{0}(\bar{\omega})\right|^{2}\right\rangle|\tilde{G}(\bar{z}, \bar{\omega})|^{2},
\end{aligned}
$$

where the angular brackets denote the ensemble average, $E_{L}=P_{\text {ave }} / \rho P_{\text {beam }}$, where $P_{\text {ave }}$ is the average emitted power and $P_{\text {beam }}=m c^{2} \gamma_{0} I / e$ is the electron beam power. The last part of Eq. (6) holds in a limit of a long bunch, neglecting the contributions of Eq. (3).

As discussed previously, the function $|\tilde{G}|^{2}$ has a bandwidth $\Delta k \simeq 1 / \ell_{c}$, whereas the noise power, proportional to $\left\langle\left|\tilde{b}_{0}\right|^{2}\right\rangle$, is a superposition of random spikes with a width $\Delta k \propto 1 / \ell_{b}$ over a range of frequencies of width equal to the reciprocal of the noise correlation length, $1 / \lambda_{s}$. Since $\tilde{b}_{0}(\bar{\omega})$ is the Fourier transform of $b_{0}\left(\bar{z}_{1}\right)$, and $\left\langle\left|b_{0}\left(\bar{z}_{1}\right)\right|^{2}\right\rangle=1 / N_{\lambda}$, we have that $\Delta \bar{\omega}\left\langle\left|\tilde{b}_{0}(\bar{\omega})\right|^{2}\right\rangle \simeq \bar{\ell}_{b} / N_{\lambda}$.

In the high-gain long bunch regime, $\ell_{b} \gg 2 \pi \ell_{c}$, the bandwidth of $\tilde{G}$ contains many random spikes; we can assume $\Delta k=1 / \lambda_{s}$ (i.e., $\Delta \bar{\omega}=1 / 2 \rho$ ) and evaluate (6):

$$
E_{L}(\bar{z})=\frac{1}{3 \sqrt{4 \pi \sqrt{3} \bar{z}} N_{\ell_{c}}} e^{\sqrt{3} \bar{z}}
$$

where $N_{\ell_{c}}=N_{\lambda}\left(\ell_{c} / \lambda_{s}\right)$ is the number of electrons in a cooperation length $\ell_{c}$. Expression (7) agrees with the results derived previously $[3,4]$ for continuous electron beams. Since $b_{0}\left(\bar{z}_{1}\right)$ can be thought of, in the long bunch case, as a sum of random delta functions and $G\left(\bar{z}, \bar{z}_{1}\right)$ has a full temporal width of the order of $2 \pi \ell_{c}$, Eq. (2) states that the temporal structure of the radiation pulse is a random superposition of many spikes, with a maximum peak to peak distance $2 \pi \ell_{c}$. These results are obtained also from the numerical model, as shown in Figs. 1(b) and $1(\mathrm{c})$.

From (7) it is possible to evaluate the saturation length of $E_{L}$. Since, in the long bunch case, $E_{L}$ at the usual steady-state saturation reaches a value close to 1 , i.e., $P_{\text {ave }} \simeq \rho P_{\text {beam }}$, for $N_{w} \simeq 1 / \rho$, or, in scaled units, for $\bar{z}=4 \pi$, from Eq. (7) we can evaluate the saturation length as $\bar{z}_{s} \simeq \frac{1}{\sqrt{3}} \ln \left[12 \pi 3^{1 / 4} N_{\ell c}\right] \simeq 2.25+\frac{1}{\sqrt{3}} \ln N_{\ell_{c}}$. This estimate is in good agreement with the simulations.

Conversely, in the short bunch case, $\ell_{b} \leq 2 \pi \ell_{c}$, it is possible to neglect the radiation inside the bunch, due to the strong slippage, and it is easy to see that the field given by Eq. (3) is a smooth function of $\bar{z}_{1}$, since the randomness of $b_{0}$ has been integrated over the bunch. It follows that a single smooth superradiant pulse is emitted, as can be observed in Fig. 1(a). To evaluate expression (6) in this limit, we neglect again the inner part of the field, (2), with respect to the part of the field propagated 

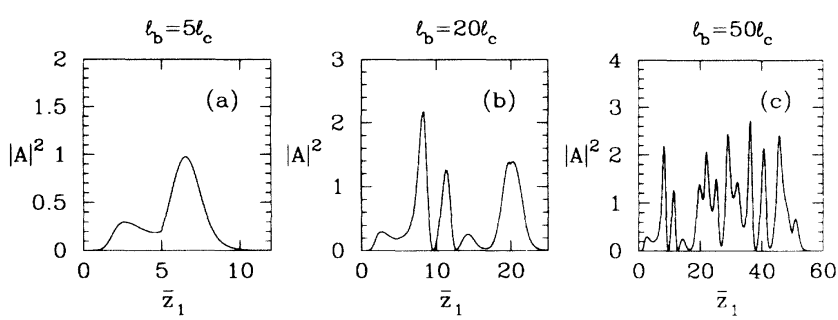

FIG. 1. Results of the numerical model: temporal structure of the radiated pulse, $|A|^{2}$ vs $\bar{z}_{1}$, at the first saturation, for three values of the electron bunch length, at $z=14 \ell_{g}$ and for $\left\langle\left|b_{0}\right|^{2}\right\rangle=10^{-6}$ : (a) $\ell_{b}=5 \ell_{c}$, (b) $\ell_{b}=20 \ell_{c}$, and (c) $\ell_{b}=50 \ell_{c}$. The temporal scale is in units of $z_{1}=\left(z-v_{\|} t\right) / \ell_{c}$.

out of the leading edge, (3). An asymptotic evaluation of $E_{L}$ leads to the following approximated expression:

$$
E_{L}(\bar{z})=\frac{\sqrt{\bar{\ell}_{b}}}{12 \pi \sqrt{3} N_{\lambda}} \frac{e^{3 \sqrt{3}(y / 2)^{2 / 3}}}{(y / 2)^{5 / 3}},
$$

where $y=\sqrt{\bar{\ell}_{b}} \bar{z}$. We note that $E_{L}$ does not experience an exponential growth with respect to $\bar{z}$ and that the growth rate depends on the bunch length as $\sqrt{\bar{\ell}_{b}}$.

In the short bunch limit it is possible to demonstrate [8] that, at saturation, $P_{\text {ave }} \simeq \rho P_{\text {beam }} \sqrt{\ell_{b} / \ell_{c}} \propto n_{e}^{3 / 2}$. This can be shown analytically from the superradiant self-similar solution, described in Ref. [8], and by the following intuitive argument: The average power can be approximated by the product of the peak power, $|A|^{2} \propto$ $\left(\ell_{b} / \ell_{c}\right)^{2}$, and the width, $\Delta \bar{z}_{1} \propto\left(\ell_{c} / \ell_{b}\right)^{1 / 2}$, divided by the scaled bunch length $\ell_{b} / \ell_{c}$, so that $E_{L} \simeq \sqrt{\ell_{b} / \ell_{c}}$. From (8), imposing that $E_{L}=P_{\text {ave }} / \rho P_{\text {beam }} \simeq \sqrt{\ell_{b} / \ell_{c}}$ we can see that the saturation length scales as $\bar{z}_{s} \simeq \sqrt{\ell_{c} / \ell_{b}}$.

In the long bunch case, after the usual steady-state saturation ( $\left.E_{L} \simeq 1\right), E_{L}$ continues to increase (see Fig. 2), due to the growth of the superradiant spikes emitted by the electron bunch, up to a second saturation value. This second saturation value scales, as in the short bunch case, as $n_{e}^{3 / 2}$, since the peak intensity of the spikes is proportional to $n_{e}^{2}$ and their time duration is proportional to $1 / \sqrt{n_{e}}[8]$.

We have used the 1D time dependent numerical model previously employed for the study of superradiance in the free-electron laser (see Ref. [10]), where the proper slippage between the electron bunch and the radiation pulse is taken into account. The electron beam is "sampled" at each radiation wavelength, where the shot noise in the electron phases is generated through a simple algorithm. The simulation electrons are first spaced uniformly along the radiation wavelength; then their position is perturbed by a small random amount, distributed with a Gaussian probability of width $\Delta[11,12]$. The parameter $\Delta$ is determined by the requirement on the initial average bunching along the electron beam, $\left\langle\left|b_{0}\right|^{2}\right\rangle$. Imposing that $\left\langle\left|b_{0}\right|^{2}\right\rangle \simeq 1 / N_{\lambda}$, the parameter $\Delta$ turns out to be $\Delta \simeq \sqrt{n / N_{\lambda}}$, where $n$ is the number of simulation electrons in each radiation wavelength.

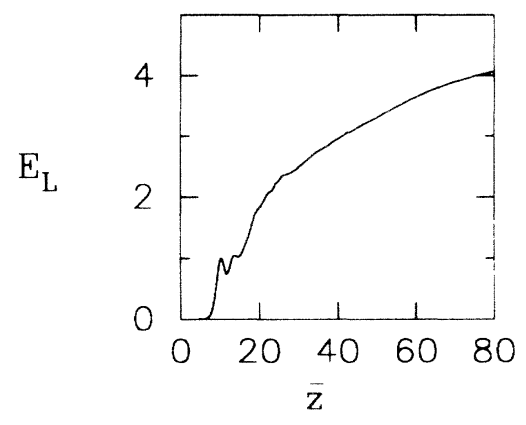

FIG. 2. Long bunch case, $\ell_{b}=100 \ell_{c}$. Average emitted power as a function of the dimensionless distance along the wiggler, $\bar{z}=z / \ell_{g}$, showing the first and second saturation values.

We consider the long pulse limit. In the time domain, the noisy pattern of the electron bunching along the beam leads to a superradiant spiking in the radiation pulse. The initial irregular spiking, seeded by nonuniformities on the scale of a radiation wavelength, cleans up and tends to a more regular pattern, with the occurrence of one spike every $2 \pi$ cooperation lengths. Hence, due to this "intrinsic distance" of the superradiant spikes, no more than one spike can develop every $2 \pi \ell_{c}$, as shown in Fig. 1 for three different pulse lengths. The position of the spikes, however, is random and depends strongly on the initial noise pattern $b_{0}\left(\bar{z}_{1}\right)$. Each spike exhibits the superradiant scaling of intensity as the square density of the electron beam, as has been numerically tested. If the pulse is shorter than or of the order of $2 \pi \ell_{c}$, only one "clean" spike occurs, as can be seen in Figs. 1 and 3.

For small undulator lengths we have found that the spectral bandwidth shrinks as $1 / N_{w}$, in agreement with the undulator radiation spectrum width [13]. In the highgain region the envelope reaches the well known bandwidth $[3,5] \Delta k / k_{s} \simeq 2 \rho$, i.e., $\Delta k \simeq 2 k_{s} \rho=1 / \ell_{c}$, as one expects from the Fourier transform of the temporal structure described above. If $\ell_{b} \leq 2 \pi \ell_{c}$, the spectrum is composed by a single line, as shown in Fig. 3(a).

Only the smooth envelope has been the object of most of the previous theories (see Refs. [3,5]), even if a preliminary work on the frequency spiking for infinite electron bunches can be found in Ref. [14]. In order to model correctly this spiking behavior, it is necessary to take

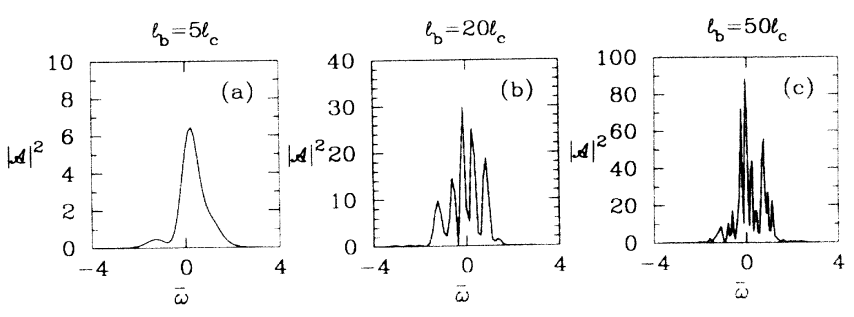

FIG. 3. Spectrum of the radiated pulses, for the same cases of Fig. 1 ; here $\vec{\omega}=\Delta \omega / 2 \rho \omega$. 


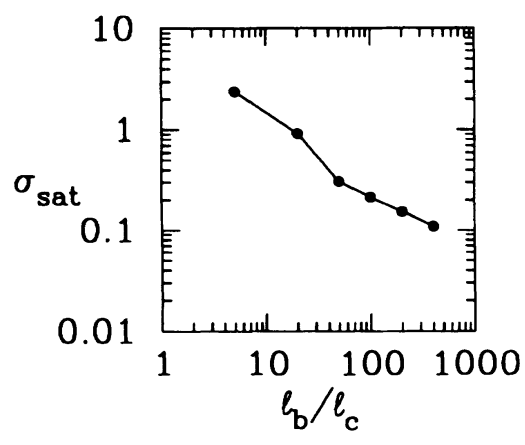

FIG. 4. Shot to shot fluctuations. Fluctuation of the saturation length as a function of $1 / \sqrt{\ell_{b} / \ell_{c}}$.

properly into account not only the slippage between the radiation and electron pulses, but also the finite bunch length, which implies the cleaning up of the pulses.

$E_{L}$ (the average emitted power over the whole radiation pulse) grows exponentially along the wiggler, up to a saturation value near to the usual $\rho P_{\text {beam }}$, as can be seen in Fig. 2. However, after this first saturation, unlike the previous theories, it continues to increase up to a second saturation value, even for a long bunch. This behavior can be explained qualitatively by the superradiant character of the spiking starting from noise. In fact, as discussed in Ref. [9], a single spike slipping over fresh electrons continues to grow and narrow without trapping the electrons and extracting energy from them with large efficiency. However, the superradiant spikes generated from noise stop growing when they slip over electrons that have already interacted with previous radiation spikes that have left them with a large amount of energy spread.

Our numerical analysis allows us to make an estimate of the shot to shot fluctuations in the initial electron bunching on the saturation length for $E_{L}$. In particular, we kept the same average initial value of the bunching on the initial pulse for a series of simulations performed with different random seeds. Since the superradiant evolution has a "coherence length" given by $\ell_{c}, E_{L}$ can be thought of as an average over $\ell_{b} / \ell_{c}$ statistically independent processes. With this statistical assumption the fluctuations in the physically interesting quantities, as the saturation length and the saturated power, should depend as $1 / \sqrt{\ell_{b} / \ell_{c}}$, i.e., as the inverse square root of the statistically independent regions of the electron bunch. A numerical test has confirmed this dependence and is shown in Fig. 4 for the fluctuations in the saturation length. Each point of this figure indicates the amplitude of rms fluctuations relative to a series of 100 runs with a given beam length, but different seeds for the random number generator. Because of this fact the shot to shot fluctuations can vary from several gain lengths in the short bunch regime to a fraction of a gain length in the long bunch regime. This issue may prove important for the design of future RF linac based $x$ ray FELs. The current parameters for the SLAC Collaboration range from a value of $\ell_{b} / \ell_{c} \simeq 400$ at $4 \mathrm{~nm}$ to a value smaller than 10 for the proposed test facility at 80-240 nm.

The shot to shot fluctuations in each one of the statistically independent regions of the electron beam, in the exponential regime, can be easily interpreted in terms of the fluctuations in the initial noise power $P_{0} \propto\left|b_{0}\right|^{2}$. Since we have assumed a Gaussian distribution, the relative fluctuation $\delta P_{0} / P_{0}$ will be approximately equal to 1 . From this fact and the exponential law $P(\bar{z})=$ $P_{0} \exp (\sqrt{3} \bar{z})$, it follows that the emitted power fluctuation $\delta P / P$ is of the order of 1 . Furthermore, $\delta P_{0} / P_{0} \simeq 1$ implies $\delta \bar{z}_{\text {sat }} \simeq 1$, or $\delta z_{\text {sat }} \simeq \ell_{g}$; that is, the saturation length fluctuations for each statistically independent portion of the bunch are of the order of the gain length.

In this Letter we have used a model to describe the FEL startup process from the random noise in the particle phases. The main characteristics of the emitted radiation, in both the time and frequency domains, are analyzed for the case of short and long (with respect to the cooperation length) electron bunches. In both cases the system exhibits a superradiant evolution seeded by the shot noise nonuniformities of the electron beam. The usual steady-state behavior for long bunches is shown only by the average emitted power, whereas the structure of the pulses is dominated by a random pattern of superradiant spikes. An analysis of the shot to shot fluctuations of the output power and saturation length shows that the fluctuations tend to be small in the long bunch regime, whereas they can be huge in the short pulse case, reaching the same order of magnitude of the average value of the observable.

[1] R. Bonifacio, C. Pellegrini, and L. Narducci, Opt. Commun. 50, 313 (1984).

[2] C. Pellegrini et al., Nucl. Instrum. Methods Phys. Res., Sect. A 331, 223 (1993).

[3] J.M. Wang and L.H. Yu, Nucl. Instrum. Methods Phys. Res., Sect. A 250, 484 (1986).

[4] K.J. Kim, Nucl. Instrum. Methods Phys. Res., Sect. A 250, 396 (1986).

[5] K.J. Kim, Phys. Rev. Lett. 57, 1871 (1986).

[6] W.M. Fawley, A.M. Sessler, and E.T. Scharlemann, in Proceedings of the 1993 Particle Accelerator Conference, Washington, DC (to be published).

[7] R. Bonifacio, C. Maroli, and N. Piovella, Opt. Commun. 68, 369 (1988).

[8] R. Bonifacio, L. De Salvo, P. Pierini, and N. Piovella, Nucl. Instrum. Methods Phys. Res., Sect. A 296, 358 (1990).

[9] R. Bonifacio, N. Piovella, and B.W.J. McNeil, Phys. Rev. A 44, 3441 (1991).

[10] R. Bonifacio, B.W.J. McNeil, and P. Pierini, Phys. Rev. A 40, 4467 (1989).

[11] R. Graham and S. Isermann, Phys. Rev. A 43, 3982 (1991).

[12] C. Penman and B.W.J. McNeil, Opt. Commun. 90, 82 (1992).

[13] D.F. Alferov et al., Sov. Phys. Tech. Phys. 18, 1336 (1974).

[14] C. Penman, Opt. Commun. 91, 377 (1992). 